\begin{tabular}{|l|l|l|l|l|}
\hline Cuadernos I. Geográfica & 24 & pp. 89-106 & Logroño & 1998 \\
\hline
\end{tabular}

\title{
EL MICROCLIMA URBANO DE LOGROÑO EN INVIERNO: UN MODELO ESPACIAL DE COMPORTAMIENTO HIGROTÉRMICO
}

\author{
L. ORTIGOSA IZQUIERDO' \\ I. SOBRÓN GARCÍA ${ }^{2}$ \\ A. GÓMEZ VILLAR ${ }^{3}$
}

\begin{abstract}
RESUMEN. Se estudia el comportamiento bigrotérmico invernal en la ciudad de Logroño -ciudad de tamaño medio-, a partir de muestreos en trayectos preseleccionados en diferentes dias $y$ boras. El análisis de los datos permite cuantificar la intensidad de la "isla de calor" en el centro urbano, cuya diferencia térmica con el entorno rururbano no excede nunca los $3^{\circ} \mathrm{C}$ (promedio $1.7^{\circ} \mathrm{C}$ ). Se observa que el efecto térmico de la ciudad no responde a un modelo central. La ciudad muestra áreas relativamente más cálidas y frías en función de la importancia de la red viaria, la estructura de las edificaciones y los usos del suelo. El confort climático derivado de la bumedad relativa también es muy contrastado, sobre todo por la influencia del río Ebro. Empleando un SIG, se elabora mediante interpolación espacial un modelo cartográfico del efecto calórico de la ciudad y su entorno, que refleja una distribución multinúcleo.
\end{abstract}

ABSTRACT. In this paper the winter temperature and bumidity in Logroño (Spain) is studied, using data of different streets, squares and routes of the city. The analysis of the information permits to evaluate the intensity of urban beat island that shows a thermic difference of $3^{\circ} \mathrm{C}$ between the city centre and the outskirts

1 Area de Geografía Física (DCHS).Universidad de La Rioja. Logroño

2 Consejería de Educación, Cultura, Juventud y Deportes. Gobierno de La Rioja

3 Departamento de Geografia. Facultad de Filosofia y Letras. Universidad de León. León 
(mean $1.7^{\circ} \mathrm{C}$ ). The authors have observed that the urban heat island does not respond to a central model. Logroño has bot or cold areas in relation to great routes, building desing and urban activities. The humidity is very conditioned by the proximity of the Ebro river. Using a GIS, a cartographic model of the urban beat island has been drawn.

Palabras clave: isla de calor urbano, temperatura, humedad, SIG, Logroño Key words: urban heat island, temperature, humidity, GIS, Logroño

\section{Introducción}

El especial comportamiento climático de las ciudades con respecto a su entorno próximo es apreciable tanto en la época estival como en la invernal. En ambas estaciones las áreas metropolitanas acusan un calentamiento apreciable, puesto que actúan como "islas de calor" provocadas, entre múltiples razones, por la baja conductividad de las superficies, el consumo y las pérdidas energéticas urbanas, etc. (Lowry, 1975). El efecto microclimático urbano es bien conocido y desde hace décadas han proliferado los estudios referentes a las grandes ciudades, como es el caso de Madrid (López Gómez, 1988, 1993), Barcelona (Martín Vide, 1987; Carreras et al, 1990, Moreno García, 1993) o Zaragoza (Cuadrat et al, 1993a; López Martín, 1995; Riva et al, 1997). En los últimos años, diversos estudios han mostrado que la distribución microclimática de los grandes espacios urbanos se aleja de los modelos concéntricos, con máxima térmica en el centro y descenso paulatino hacia la periferia. Por el contrario, la diferenciación térmica parece presentar una organización espacial dispersa, vinculada a factores tales como la estructura urbana (Bello, 1994), la distribución de las zonas verdes (Almendros, 1992) o las concentraciones industriales y residenciales. A escala temporal, el microclima urbano también está en relación con la estacionalidad (Fernández García, 1990) y los distintos ritmos diarios (San Pedro, 1994).

En el caso de las pequeñas y medianas ciudades, los estudios microclimáticos han sido menos frecuentes. No obstante, se ha comprobado en ciudades como Teruel (Cuadrat et al, 1993b), Tarragona (Brunet, 1992), San Sebastián (Goiketxea, 1991) o Logroño (García Ruiz et al, 1989) que también existe un efecto de diferenciación térmica a nivel del suelo que depende de determinados factores.

Este estudio se ha llevado a cabo en Logroño, ciudad emplazada a orillas del río Ebro, a una altitud de 384 m.s.n.m (Latitud: $42^{\circ} 28^{\prime} \mathrm{N}$; Longitud: $2^{\circ} 27^{\prime} \mathrm{O}$ ). 
Se extiende por la margen derecha del río y ocupa un espacio llano correspondiente a terrazas fluviales (Fig.1). Sigue un modelo de crecimiento semiconcéntrico hacia el sur, oeste y este, actuando el río Ebro, por el norte, de barrera a la expansión urbana. La ciudad acoge a 126.000 habitantes, concentrados en un núcleo urbano relativamente bien definido por las edificaciones en altura. De aquí surgen cinco importantes vías de comunicación en torno a las cuales se articula el cinturón periurbano. Dentro del mismo se incluyen, al menos, dos poblaciones: Villamediana y Lardero, con autonomía administrativa pero inmersas en el área de influencia de Logroño.

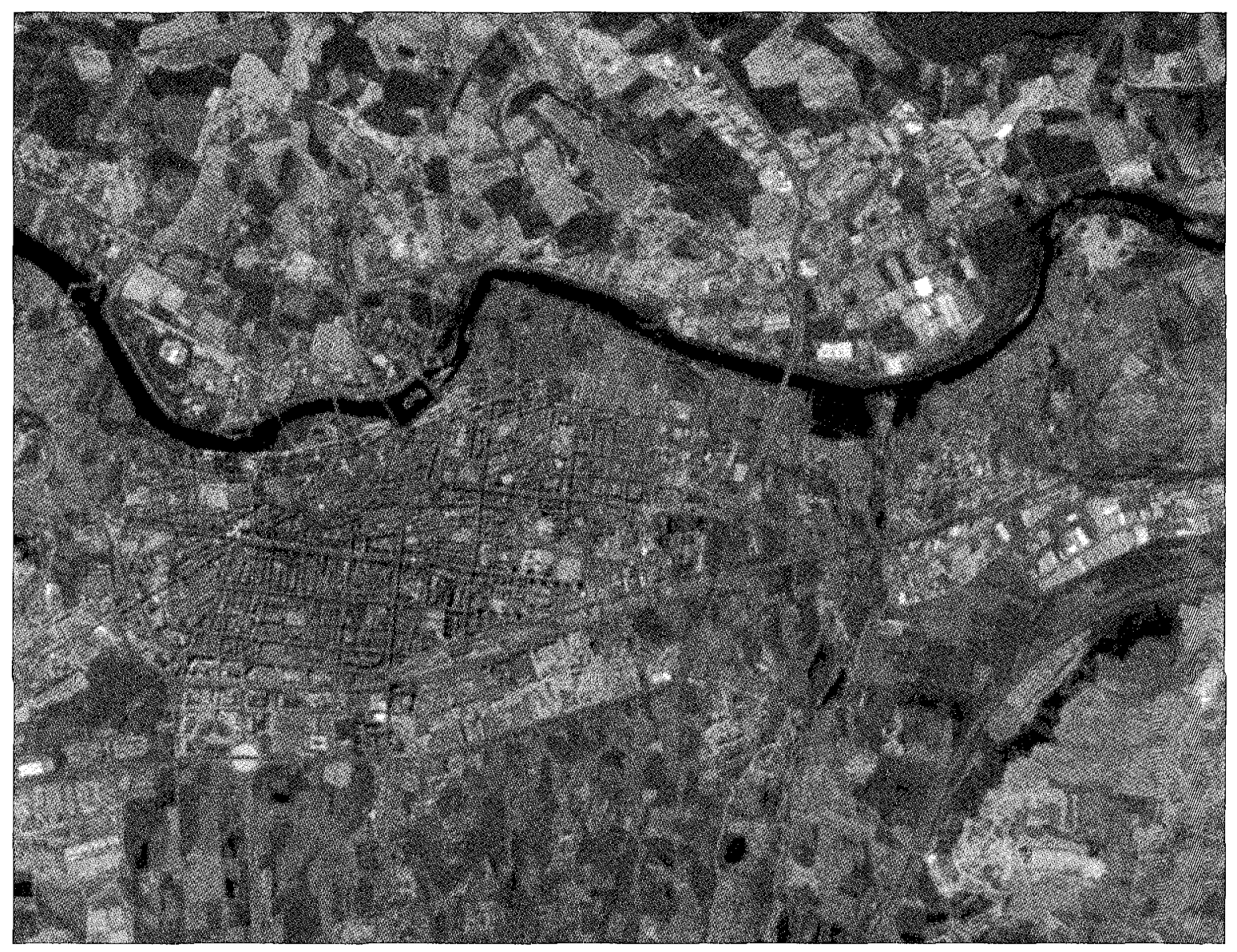

Fig.1 Imagen aérea de la ciudad de Logroño

El clima general de la ciudad es mediterráneo con matices continentales (Sánchez Gabriel y Fernández Giró, 1979; Nuñez Olivera y Martínez Abaigar, 1991). El primer estudio sobre el efecto "isla de calor" en Logroño fue realizado por García-Ruiz y colaboradores en 1989, revelando la existencia de un contraste térmico entre el exterior e interior de la ciudad que se manifiesta en todas las estaciones y tipos de tiempo. No obstante, las diferencias térmicas 
registradas fueron muy discretas, nunca superiores a $3^{\circ} \mathrm{C}$. Dicho estudio evidenció también que el calentamiento urbano se comporta de modo diferente en las estaciones del año. Durante el invierno, caracterizado por bajas temperaturas y menos horas de sol, el efecto calórico de la urbe es menos apreciable. En dicha estación las diferenciaciones térmicas surgen vinculadas al consumo energético. Durante el verano, caluroso y soleado, el fuerte efecto de irradiación de las superficies urbanas acentúan el pico de calor de la ciudad con respecto a su entorno, de modo que a las 23 horas se aprecian las diferencias térmicas más contrastadas.

Siguiendo esta línea de investigación, se pretende, en primer lugar, ampliar la información sobre el comportamiento térmico e higrométrico en la ciudad de Logroño y su entorno durante los meses invernales. Empleando el método de los trayectos urbanos (López Gómez, 1988), se ha tomado en esta ocasión un mayor número de puntos de observación. En segundo lugar, y a partir de un tratamiento estadístico y gráfico, basado en un Sistema de Información Geográfica (Grimmond \& Souch, 1994), se ha diseñado un modelo espacial de comportamiento higrotérmico de la ciudad de Logroño.

\section{Métodos}

Durante el invierno de 1996 se recogieron datos higrotérmicos en la ciudad de Logroño en diferentes días y horas siguiendo el método de los transectos o recorridos urbanos. Exactamente, los muestreos se realizaron en tres trazados diferentes: un circuito urbano con vehículo, el centro (espacios verdes y casco antiguo peatonal) y la periferia urbana. En estos tres trazados, se completaron 21 muestreos a diferentes horas: 7-8 de la mañana, 2-3 de la tarde y 10-11 de la noche. En total se dispuso de información de 83 puntos (Fig. 2). La relación de puntos de muestreo se apoyó en los siguientes criterios básicos:

- Consideración de la diversidad urbana (grandes vías de tránsito, calles secundarias en manzanas residenciales, parques, casco antiguo, ...)

- Localización en relación a la centralidad

- Proximidad de los puntos de muestreo para que el trabajo de campo pudiera realizarse en el menor tiempo posible (una hora como máximo).

En la tabla 1 se puede comprobar la relación completa de los 83 puntos considerados $y$, como ejemplo, los resultados obtenidos en una determinada fecha y hora. La medición en cada punto seleccionado se realizó con un higrotermómetro digital de precisión (errores relativos de $0.1{ }^{\circ} \mathrm{C}$ y $1 \%$ en la escala de humedad), asegurándonos de medir los parámetros de temperatura, humedad relativa y punto de rocío a una altura del suelo de $1.5 \mathrm{~m}$ (estándar meteorológico). 


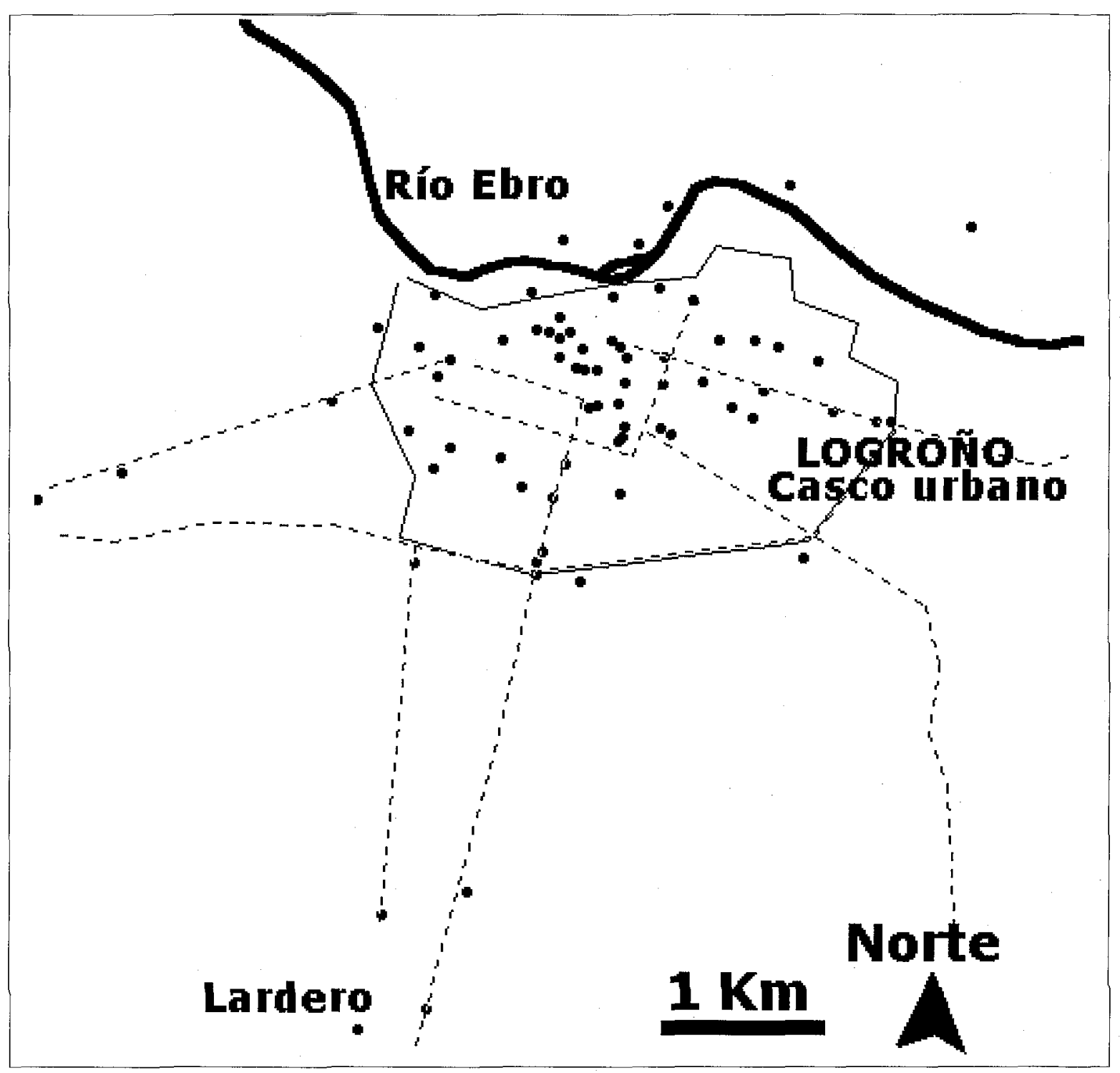

Fig. 2 Puntos seleccionados para la recogida de datos

La metodología empleada en el trabajo aporta una gran fiabilidad en las mediciones parciales, aunque impide medir todos los puntos seleccionados en el mismo instante y con los mismos instrumentos; lógicamente, solo se puede tomar una medición en un tiempo y lugar, lo cual considerando la dinámica higrotérmica representa un grave inconveniente para el tratamiento de los datos. Por ello, se seleccionaron unos puntos de control comunes en los diferentes trazados muestrales que nos permitieron corregir en gran parte dicho problema. Gracias a los puntos de control se ha comprobado que a lo largo de los 60 minutos de cada muestreo se dan ciertas diferencias térmicas para un mismo punto de la ciudad, cuyo máximo observado ha llegado a ser de $0.5^{\circ} \mathrm{C}$. 
Tabla 1. Puntos de muestreo considerados y ejemplos de comportamiento higrotérmico invernal en los tres trazados

CIUDAD 31/1/96, Hora 8 a.m.

\begin{tabular}{|c|c|c|c|}
\hline PUNTOS DE MUESTREO & $\begin{array}{c}\text { Temp. } \\
\mathrm{T}^{\circ}{ }^{\circ} \mathrm{C}\end{array}$ & $\begin{array}{l}\text { Humd. } \\
\text { Rel.\% }\end{array}$ & $\begin{array}{c}\text { Rocío } \\
\mathrm{T}^{\circ} \mathrm{C}\end{array}$ \\
\hline Jorge Vigón 46 & 8.2 & 94.7 & 7.4 \\
\hline Hospital S. Millán & 8.2 & 94.7 & 7.4 \\
\hline $\mathrm{Paz}$ & 8.2 & 95.4 & 7.5 \\
\hline Seminario & 8.1 & 95.9 & 7.6 \\
\hline Rotonda (fin Avda. Paz) & 7.8 & 96.7 & 7.3 \\
\hline S.J. Calasanz/Luis Ulloa & 8.3 & 96.1 & 7.7 \\
\hline S.J. Calasanz/Cameros & 8.2 & 96.3 & 7.6 \\
\hline San Millán & 8.1 & 96.0 & 7.6 \\
\hline S. Ascarza/Escuelas Pías & 8.2 & 95.9 & 7.6 \\
\hline Plaza de Toros & 8.3 & 95.9 & 7.7 \\
\hline P. Constitución (Parque Ebro) & 8.3 & 96.3 & 8.0 \\
\hline Puente de Piedra (m. dercha.) & 8.4 & 95.9 & 7.7 \\
\hline Puente de Hierro (m. izqda) & 8.2 & 96.0 & 7.9 \\
\hline Puente Hierro /Sagasta & 8.4 & 94.7 & 7.6 \\
\hline P.C. Espolón/Sagasta & 8.8 & 92.8 & 7.8 \\
\hline Rotonda Murrieta & 8.7 & 93.8 & 7.8 \\
\hline Escuela en Carret. El Cortijo & 8.4 & 95.3 & 7.8 \\
\hline Poniente /Gonzalo Berceo & 8.7 & 92.9 & 7.7 \\
\hline Gonzalo Berceo/La Campa & 8.8 & 89.6 & 8.0 \\
\hline P.C. Gonzalo Berceo/M. Murrieta & 8.7 & 93.0 & 7.7 \\
\hline Rey Pastor & 8.7 & 93.7 & 7.8 \\
\hline Rey Pastor/Huesca & 8.7 & 92.8 & 7.7 \\
\hline Huesca/Chile & 9.1 & 92.1 & 7.9 \\
\hline Plazuela Acesur/Múgica & 8.9 & 92.4 & 7.8 \\
\hline Somosierra/Rep. Argentina & 8.8 & 89.5 & 8.2 \\
\hline Vara de Rey/Somosierra & 8.6 & 94.3 & 7.6 \\
\hline Rotonda(V.Rey/D. de Nájera) & 8.4 & 93.0 & 7.8 \\
\hline Estación de ferrocarril & 8.5 & 95.0 & 7.6 \\
\hline Jorge Vigón /Marqués Ensenada & 8.9 & 92.8 & 7.7 \\
\hline P.C. Jorge Vigón/Carmelitas & 9.1 & 91.3 & 7.8 \\
\hline Comun. Autónoma (Vara de Rey) & 8.6 & 92.8 & 7.8 \\
\hline Muro Carmen/Muro Cervantes & 8.9 & 94.4 & 7.8 \\
\hline Avda. Paz (Ayuntamiento) & 8.9 & 93.1 & 8.1 \\
\hline Calvo Sotelo/Avenida Colón & 9.1 & 91.9 & 7.9 \\
\hline Duquesa Victoria (Polideportivo) & 8.9 & 93.6 & 8.0 \\
\hline Padre Claret/Nuestra. Sra. Paz & 8.5 & 91.6 & 8.2 \\
\hline
\end{tabular}

CIUDAD

31/1/96, Hora 11.30 p.m.

\begin{tabular}{lccc}
\hline PUNTOS DE MUESTREO & $\begin{array}{c}\text { Temp. } \\
\mathrm{T}^{\circ} \mathrm{C}\end{array}$ & $\begin{array}{c}\text { Humd. } \\
\text { Rel.\% }\end{array}$ & $\begin{array}{c}\text { Rocío } \\
\mathrm{T}^{\circ} \mathrm{C}\end{array}$ \\
\cline { 1 - 3 } Parque El Carmen centro & 9.9 & 88.3 & 8.0 \\
Parque El Carmen medio & 10.2 & 83.8 & 7.9
\end{tabular}




\begin{tabular}{|c|c|c|c|}
\hline P. El Carmen/C. Villamediana & 10.4 & 83.8 & 7.9 \\
\hline Jorge Vigón/Carmelitas & 11.0 & 82.3 & 8.2 \\
\hline Estatua Labrador (Jorge Vigón) & 10.2 & 86.6 & 8.2 \\
\hline Dctres Castroviejo/Juan XXIII & 10.6 & 83.5 & 8.3 \\
\hline Juan XXIII/Calvo Sotelo & 10.4 & 87.4 & 8.3 \\
\hline Comun. Autónoma (Vara de Rey) & 10.5 & 86.5 & 8.3 \\
\hline Espolón medio & 10.6 & 86.0 & 8.3 \\
\hline Espolón centro & 10.4 & 85.9 & 8.1 \\
\hline Sagasta/Bretón de los Herreros & 10.2 & 86.6 & 8.0 \\
\hline Fuente Murrieta & 10.6 & 86.6 & 8.3 \\
\hline Merced (Biblioteca Pública) & 11.1 & 83.5 & 8.5 \\
\hline Marqués S. Nicolás/ Merced & 10.7 & 86.5 & 8.5 \\
\hline F. Martínez. Zaporta & 10.8 & 86.5 & 8.5 \\
\hline Portales (centro) & 11.0 & 84.7 & 8.5 \\
\hline Puente Hierro (Sagasta) & 11.1 & 83.6 & 8.5 \\
\hline Plaza del Mercado & 10.8 & 83.2 & 8.1 \\
\hline Calle San Juan (Samaray) & 11.1 & 83.7 & 8.5 \\
\hline Muro Carmen/Muro Cervantes & 11.7 & 79.3 & 8.2 \\
\hline Glorieta D. Zubía (centro) & 11.1 & 83.8 & 8.4 \\
\hline Glorieta D. Zubía /Juan XXIII & 10.9 & 83.4 & 8.2 \\
\hline PERIFERIA & \multicolumn{2}{|c|}{ 8/2/96, Hora 8 a.m } & \\
\hline PUNTOS DE MUESTREO & $\begin{array}{c}\text { Temp. } \\
\mathrm{T}^{\circ} \mathrm{C}\end{array}$ & $\begin{array}{c}\text { Humd. } \\
\text { Rel.\% }\end{array}$ & $\begin{array}{c}\text { Rocío } \\
\mathrm{T}^{\circ} \mathrm{C}\end{array}$ \\
\hline Vara de Rey/d. de Nájera & 6.2 & 62.4 & -0.5 \\
\hline Avda. Madrid & 6.3 & 61.8 & -0.5 \\
\hline Rotonda Avda. Madrid/Circunval. & 6.0 & 62.6 & -0.5 \\
\hline Circunvalación (sector central) & 6.0 & 61.2 & -0.7 \\
\hline Circunvalación (sector este) & 6.1 & 60.8 & -0.4 \\
\hline Rotonda Circunvalación/Avda. La Paz & 6.5 & 61.9 & -0.3 \\
\hline Rotonda Avda. Mendavia/Circunval. & 6.3 & 66.2 & 0.4 \\
\hline Carret. Mendavia (Monte Cantabria) & 6.0 & 66.4 & 0.2 \\
\hline Carret. Mendavia (Pozo Cubillas) & 5.8 & 66.3 & 0.1 \\
\hline Puente Piedra (margen izquierda) & 6.0 & 64.8 & -0.2 \\
\hline Puente Hierro (margen izquierda) & 6.0 & 67.2 & 0.2 \\
\hline Puente Hierro/Sagasta & 6.2 & 62.2 & 0.4 \\
\hline Sagasta/Bretón & 6.6 & 62.0 & -0.3 \\
\hline Fuente Murrieta & 6.2 & 62.4 & -0.4 \\
\hline Gonzalo Berceo/Marqués Murrieta & 6.6 & 61.9 & -0.3 \\
\hline M. Murrieta (Ferrocarril) & 6.3 & 62.8 & -0.7 \\
\hline Yagüe & 6.0 & 62.8 & -0.6 \\
\hline Yagüe (Ulecia) & 5.8 & 65.6 & -0.5 \\
\hline Rotonda Calle Chile & 6.3 & 66.8 & -0.5 \\
\hline Rotonda Avda. Salustiano (Circunv.) & 4.8 & 79.8 & -0.5 \\
\hline Acceso A-68 & 4.4 & 82.4 & 1.7 \\
\hline Lardero (centro pueblo) & 5.1 & 79.5 & 1.7 \\
\hline Lardero/Carretera Soria & 5.0 & 77.6 & 1.6 \\
\hline CC.EE.II. & 5.2 & 78.2 & 1.7 \\
\hline Rotonda Avda. Madrid/Circunval. 2 & 5.4 & 78.7 & 1.8 \\
\hline
\end{tabular}


Posteriormente, el conjunto de datos obtenido en todos los muestreos ha sido informatizado, con el fin de lograr síntesis cartográficas y estadísticas (medias, amplitud e índices de dispersión). Sin embargo, es importante señalar que la información cuantitativa más relevante se deduce de una homogeneización estadística de los datos muestrales, ya que -como se ha apuntado anteriormente- la variabilidad de cada muestreo -diferentes días y horas- exige una corrección de las mediciones brutas. Para este estudio, la técnica fundamentalmente empleada ha sido el manejo de los valores estandarizados de cada serie (media de 0 y desviación estándar de 1). Este método estadístico, de validez ampliamente probada (Spiegel, 1970; SPSS, 1990), ofrece la posibilidad de comparar y establecer el modelo de funcionamiento de la isla de calor urbana a partir de un SIG (Eastman, 1995).

Tabla 2 Algunas observaciones higrotérmicas en el ámbito ciudad-periferia en distintas fechas $y$ boras

\begin{tabular}{ccccccc} 
DÍA/HORA muestreo & $\begin{array}{c}\text { Media } \\
\text { aritmética }\end{array}$ & $\begin{array}{c}\text { Desviación } \\
\text { estándar }\end{array}$ & Máxima & Valor & Diferencia & $\begin{array}{c}\text { Coef. } \\
\text { Mínima }\end{array}$ \\
\hline
\end{tabular}

2/2/96 Hora 2.30 p.m

$\begin{array}{lrrrrrr}\text { Temperatura } \mathrm{T}^{\circ} \mathrm{C} & 13.19 & 0.64 & 15.10 & 12.30 & 2.80 & 4.88 \\ \text { Humedad rel. \% } & 52.91 & 2.72 & 57.80 & 46.20 & 11.60 & 5.14 \\ \text { P.rocío T }{ }^{\circ} \mathrm{C} & 3.82 & 0.47 & 4.70 & 2.70 & 2.00 & 12.35\end{array}$

\section{8/2/96 Hora 8 a.m}

Temperatura $\mathrm{T}^{\circ} \mathrm{C}$

Humedad rel. \%

P.rocío $\mathrm{T}^{\circ} \mathrm{C}$

\section{1/1/96 Hora 8 p.m}

\section{Temperatura $\mathrm{T}^{\circ} \mathrm{C}$}

Humedad rel. \%

P.rocío $\mathrm{T}^{\circ} \mathrm{C}$

$\begin{array}{rrrrrr}5.88 & 0.58 & 6.60 & 4.40 & 2.20 & 9.82 \\ 67.37 & 7.17 & 82.40 & 60.80 & 21.60 & 10.64 \\ 0.08 & 0.87 & 1.80 & -0.70 & 2.50 & 1035.94\end{array}$

2/2/96 Hora 8 a.m

Temperatura $\mathrm{T}^{\circ} \mathrm{C}$

Humedad rel. \%

P.rocío $\mathrm{T}^{\circ} \mathrm{C}$

$\begin{array}{rrrrrr}9.93 & 0.27 & 10.50 & 9.50 & 1.00 & 2.73 \\ 74.95 & 2.63 & 79.70 & 71.30 & 8.40 & 3.50 \\ 5.66 & 0.40 & 6.30 & 5.10 & 1.20 & 7.04\end{array}$

$\begin{array}{rrrrrr}1.62 & 0.59 & 2.70 & 0.60 & 2.10 & 36.73 \\ 93.18 & 2.10 & 96.40 & 89.40 & 7.00 & 2.25 \\ 0.76 & 0.44 & 1.70 & 0.10 & 1.60 & 57.60\end{array}$


EL MICROCLIMA URBANO DE LOGROÑO EN INVIERNO

16/2/96 Hora 8 a.m.

$\begin{array}{lrrrrrr}\text { Temperatura } \mathrm{T}^{\circ} \mathrm{C} & 2.49 & 0.38 & 3.30 & 1.60 & 1.70 & 15.23 \\ \text { Humedad rel. \% } & 71.96 & 2.11 & 77.70 & 66.30 & 11.40 & 2.93 \\ \text { P.rocío } \mathrm{T}^{\circ} \mathrm{C} & -1.81 & 0.33 & -1.10 & -2.50 & 1.40 & -17.99\end{array}$

$17 / 2 / 96$ Hora 7 a.m

$\begin{array}{lrrrrrr}\text { Temperatura } \mathrm{T}^{\circ} \mathrm{C} & 5.15 & 0.43 & 6.40 & 4.60 & 1.80 & 8.28 \\ \text { Humedad rel. \% } & 72.98 & 1.66 & 76.10 & 69.40 & 6.70 & 2.27 \\ \text { P.rocío } \mathrm{T}^{\circ} \mathrm{C} & 0.77 & 0.21 & 1.30 & 0.50 & 0.80 & 27.36\end{array}$

\section{Resultados}

Durante enero y febrero de 1996, los tipos de tiempo fueron muy variados. Hubo días afectados por masas de aire de procedencia ártica, con temperaturas diurnas en torno a $0^{\circ} \mathrm{C}$; otros soportaron el paso de borrascas atlánticas (frentes fríos con gran nubosidad y tiempo lluvioso); y finalmente, otros se caracterizaron por situaciones anticiclonales, con tiempo despejado y temperaturas diurnas superiores a los $10^{\circ} \mathrm{C}$. No es de extrañar, pues, la variabilidad existente en los datos. En la tabla 2 se presenta una selección de los muestreos realizados entre el centro urbano y la periferia, comprobando que existen diferencias significativas entre unos puntos y otros, tanto en lo que se refiere a temperaturas como a la humedad relativa. El día 2 de febrero a las 14.30 horas se observó el mayor contraste térmico: $2.8^{\circ} \mathrm{C}$. Fue una tarde con tiempo anticiclónico, con una temperatura media de $13.2^{\circ} \mathrm{C}$ y una humedad relativa media del $53 \%$, que situaba el punto de rocío a $3.8^{\circ} \mathrm{C}$. Más representativo resultó lo observado el día 16 de ese mismo mes, al amanecer. Las diferencias térmicas entre el punto más cálido del centro urbano y la periferia fueron tan solo de $1.7^{\circ} \mathrm{C}$, con variaciones en la humedad relativa de un 12\% (66-78\%). Se comprueba que el microclima creado por la ciudad de Logroño es siempre muy discreto y, sea cual sea el tipo de tiempo o la hora del día, las diferencias térmicas en el entorno metropolitano no representan más de $3^{\circ} \mathrm{C}$.

En la tabla 2 se observa también el indicador de la dispersión estadística de cada muestreo en relación con las temperaturas (coeficiente de variación de Pearson en \%). En algunos casos, dicho coeficiente es muy bajo (<5\%) lo que significa que los registros térmicos de cada serie varían muy poco con respecto a la media; por el contrario, los valores más altos señalan diferencias más acusadas entre unas y otras localizaciones. Las mayores desviaciones estadísticas (15$30 \%$ ) están siempre en relación con los días más fríos. Esto se explica por la distribución energética en la ciudad y el tipo de tiempo atmosférico. A primeras horas del día, la actividad urbana está muy discriminada: las grandes vías o 
zonas céntricas han iniciado una intensa actividad enérgetica (tráfico, iluminación, calefacciones,...) mientras que el resto de la ciudad permanece menos dinámica. En segundo lugar, se debe tener en cuenta que las bajas temperaturas invernales aparecen asociadas principalmente a los anticiclones de origen térmico, que proporcionan estabilidad a las masas de aire (ausencia de viento - limitada transferencia térmica - y cielo sin nubosidad) y acentúan la diferenciación térmica de la ciudad.

\subsection{La distribución térmica y sus pautas de comportamiento}

Aunque las diferencias térmicas entre las distintas áreas de la ciudad son difíciles de precisar porque dependen, en gran parte, de los tipos de tiempo y las horas del día, se pueden interpretar la pautas del comportamiento térmico a partir de los datos medios. En la tabla 3 se analizan las diferencias térmicas en Logroño a partir de los puntos de muestreo, fijándonos en índices tales como las medias aritméticas o los valores estándarizados. A partir de dichos datos estadísticos podemos establecer las principales características de los contrastes a nivel del suelo:

- Las zonas más cálidas de la ciudad se localizan en el centro urbano que se corresponde con el casco antiguo -de estructura muy cerrada- y los cruces viales de las grandes avenidas, muy concurridas por transeúntes y vehículos. Es importante señalar que el núcleo del casco antiguo, con ser una zona con promedios térmicos superiores a la media, no se identifica con el área más cálida de la ciudad. Son, concretamente, las calles Sagasta-Bretón de los Herreros, Calvo Sotelo-Jorge Vigón y Gran Vía-Jorge Vigón los puntos térmicamente más cálidos de la ciudad. Estas localizaciones presentan unas temperaturas entre 1.5 y $3^{\circ} \mathrm{C}$ superiores a las zonas rururbanas.

- La franja que rodea al centro urbano -caracterizada todavía por edificaciones en altura-, presenta modestas diferencias térmicas con respecto al núcleo más interno de la ciudad (entorno a $0.5^{\circ} \mathrm{C}$, por término medio). No es, pues, apreciable un gradiente de descenso térmico en función de la distancia al centro.

- La periferia urbana, considerada desde nuestra perspectiva de estudio como el espacio contiguo a la ciudad (hasta un radio de $5 \mathrm{Kms}$ ), está representada por las carreteras de salida y las zonas residenciales adyacentes. Dos hechos son constatables en este espacio: en primer lugar, se detecta un descenso térmico y, en segundo lugar, se observan muy pocas variaciones en el interior de esta zona. Según nuestros datos, desde las 
rotondas de acceso a la ciudad por el sur hasta $5 \mathrm{Km}$ más allá del límite estrictamente urbano (proximidades de Lardero), las temperaturas sólo descienden $0.4-0.5^{\circ} \mathrm{C}$. Ello significa que, al menos para ciudades pequeñas-medias sin importantes agrupaciones industriales con incidencia energética o contaminante, la isla de calor se limita exclusivamente al casco urbano densamente edificado. De hecho, se ha comprobado que la zonas residenciales del sur de la ciudad (carretera de Soria), apenas incrementan la temperatura con respecto a los espacios agrícolas próximos.

Este esquema es válido para conocer las tendencias generales. Sin embargo, un análisis detallado demuestra que en el ámbito del propio casco urbano o en el espacio rururbano existen distorsiones. Hay espacios concretos que alteran las tendencias generales mostrándose más fríos o cálidos que su entorno próximo. Un análisis detallado de los datos ha servido para establecer 4 importantes áreas con efectos higrotérmicos especiales:

1. Las zonas verdes y los espacios no edificados. Los parques y las áreas ajardinadas (Espolón, Parque Gallarza, El Carmen etc.) disponen de cubiertas vegetales que ejercen una función reguladora. Además, son espacios alejados de los focos emisores de calor (tráfico, calefacciones, etc.). Así pues, se comportan como zonas relativamente frías y húmedas. En el caso de Logroño, estos espacios son siempre de muy reducidas dimensiones $(2-10 \mathrm{Ha})$, pero aún así se han cuantificado temperaturas entre 0.2 y $0.5^{\circ} \mathrm{C}$ inferiores a las registradas en sus bordes. Los espacios no edificados (grandes aparcamientos, fincas urbanas, plazas amplias, etc.) presentan un comportamiento térmico similar al de los parques. Como ejemplo, el día 9-2-96, a las 8 horas de la mañana, el aparcamiento de la estación del ferrocarril registraba una temperatura $0.5{ }^{\circ} \mathrm{C}$ menor que la calle contigua, a pocos metros de distancia.

2. El casco antiguo. El núcleo histórico, como el de otras tantas ciudades españolas, se caracteriza por el hacinamiento de sus edificaciones y la estrechez de sus calles. Este trazado protege térmicamente el sector, de modo que estamos ante la isla de calor más homogénea de la ciudad. En sus calles la temperatura supera en cualquier tipo de tiempo invernal y en unas décimas de grado a la existente en el resto de la ciudad.

3. Las grandes avenidas. Las grandes avenidas (Gran Vía, Avda. La Paz o Vara de Rey) se comportan como corredores para las masas de aire, canalizándolas hacia el interior de la ciudad, como han señalado reiteradamente otros investigadores. Este hecho, convierte a las avenidas en puntos relativamente fríos y venteados, sobre todo durante la noche. Es frecuente que se observen diferencias térmicas superiores a $0.5^{\circ} \mathrm{C}$ entre una rotonda o avenida abierta y la calle próxima insertada en una manzana residencial. Sin embargo, estas dife- 
rencias térmicas se pueden diluir a lo largo del día, e incluso, se llega a invertir el efecto: la gran avenida es ligeramente más cálida que las calles secundarias. Tal circunstancia es debida al tráfico de vehículos y a las pérdidas energéticas de comercios y lonjas.

4. Polígonos industriales y barrios del ámbito periurbano. Estos espacios son islas de calor relativas con relación a su entorno rural. Sin duda, el carácter masivo de sus edificaciones y el consumo energético contribuyen a la elevación térmica. Como datos representativos, debemos señalar que el Polígono Industrial de Cantabria (almacenes e industria ligera para la construcción y bienes de equipo) presenta una temperatura $0.5^{\circ} \mathrm{C}$ superior a las fincas colindantes. Hemos comprobado una desviación térmica muy similar $\left(+0.6^{\circ} \mathrm{C}\right)$ en el caso del pueblo de Lardero con su entorno rural.

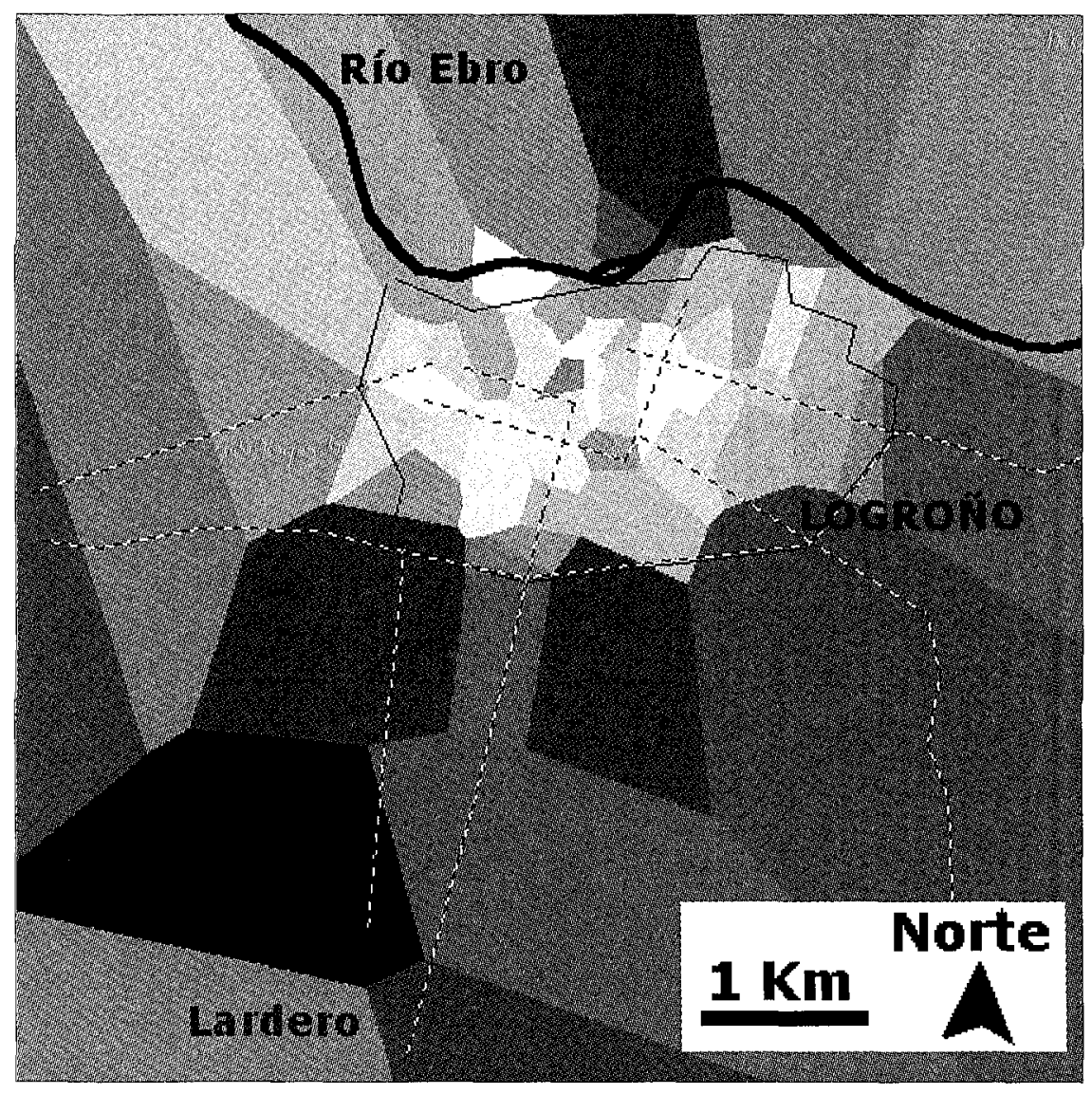

Fig.3 Distribución térmica relativa en la ciudad de Logroño interpolada según polígonos de Thiessen/Voronoi

Un modelo gráfico de la diferenciación térmica de Logroño durante el invierno puede observarse en la Figura 3. Se trata de una imagen obtenida a través de la interpolación de los valores medio muestrales (valores estandarizados) siguiendo el procedimiento de los polígonos Thiessen/Voronoi. Aunque este es un procedimiento de interpolación rudimentario, permite comprobar el desigual mosaico térmico que presenta la ciudad. Un modelo espacial más evo- 
lucionado aparece representado en la Figura 4 y corresponde a la interpolación térmica de medias estandarizadas a partir de un algoritmo que considera la ponderación de distancias entre los puntos de observación más próximos. Los tonos más claros evidencian las áreas más cálidas de la ciudad y se comprueba que la isla de calor urbana no obedece a un patrón de foco único a partir del cual se articulan sectores concéntricos térmicamente más frescos a medida que nos alejamos del centro.

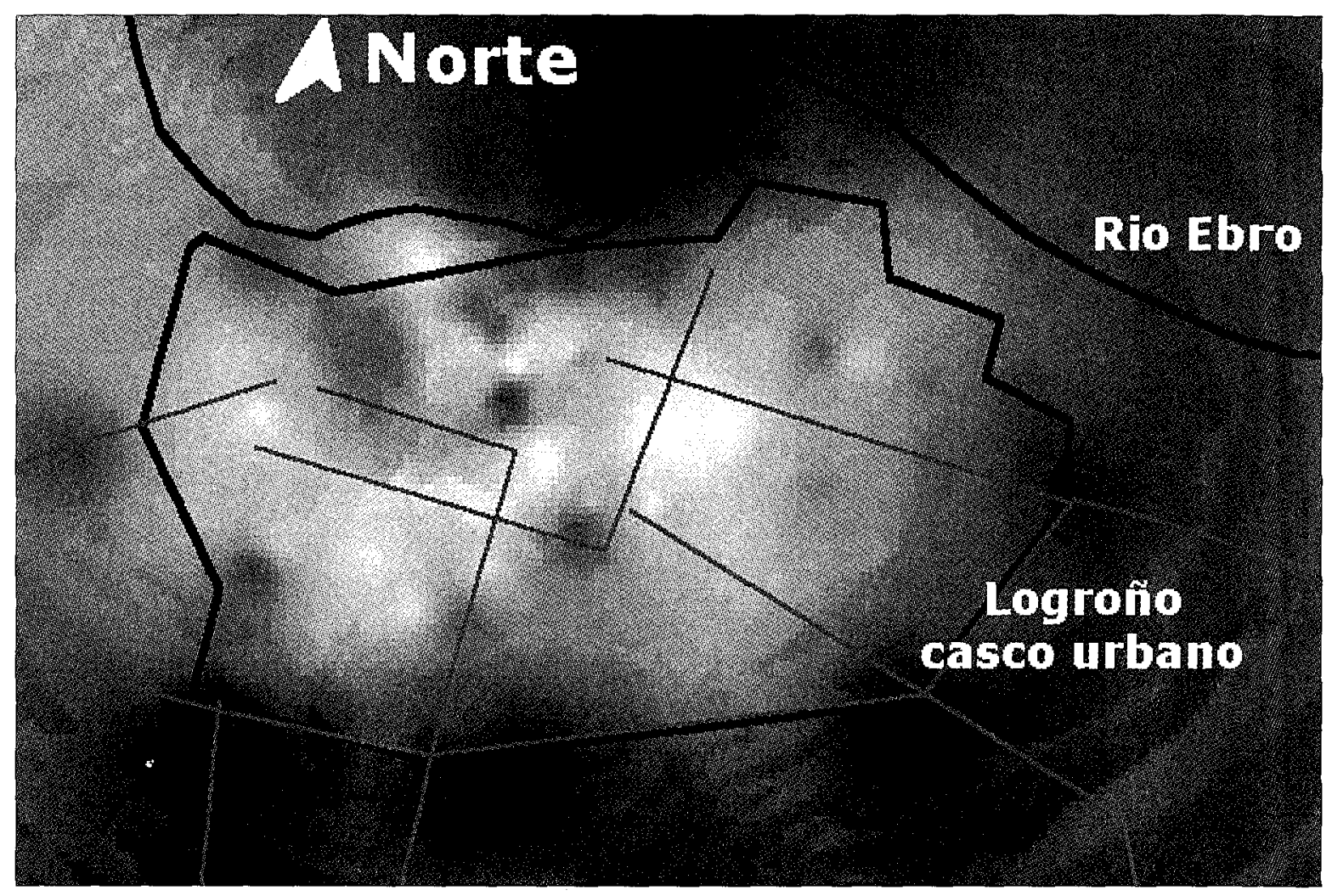

Fig.4 Modelo de la distribución térmica relativa en la ciudad de Logroño

\subsection{La bumedad y el rocío en la ciudad}

La ciudad de Logroño presenta una diferenciación microclimática ligada a la influencias húmedas del río Ebro. Por ello, es de esperar un diferente comportamiento entre la ciudad histórica, junto a la margen derecha del río, y la ciudad moderna, cada vez más alejada del eje fluvial.

Como es sabido, las superficies de agua aportan elevadas cantidades de vapor de agua a las masas de aire. Este proceso es valorado indistintamente a lo largo del año: durante el verano se considera un efecto agradable (aumenta la humedad relativa de masas de aire, muy secas e incómodas) y se aprecia negativamente en invierno, cuando los índices de humedad en el aire se apro- 
ximan muchos días a la saturación. La excesiva humedad ambiental durante la estación fría representa un inconveniente cotidiano (Fernández García, 1994), un descenso del confort que: a) acentúa el efecto de frío por la acumulación de humedad y transpiración en el vestuario personal, b) produce el rocío, especialmente en la superficies acristaladas de edificios y vehículos y c) condiciona el uso de calefacciones.

Tabla 3 Comportamiento bigrotérmico medio en las localizaciones seleccionadas

\begin{tabular}{|c|c|c|c|c|}
\hline Localizaciones & $\begin{array}{c}\text { Temperatura } \\
\text { Media (C) }\end{array}$ & $\begin{array}{l}\text { Valor Estandar } \\
\text { (Desv.St. / n) }\end{array}$ & $\begin{array}{l}\text { amedad relativa } \\
\text { Media (\%) }\end{array}$ & $\begin{array}{l}\text { V. Estandar } \\
\text { (Desv.St. / n) }\end{array}$ \\
\hline Vara de Rey/D. de Nájera & 3.98 & 0.31 & 74.05 & -0.69 \\
\hline Avda. Madrid (Asepeyo) & 4.05 & 0.45 & 73.73 & -0.80 \\
\hline Rotonda Avda. Madrid/Circunval. & 3.70 & -0.27 & 74.48 & -0.49 \\
\hline Circunvalación (sector central) & 3.40 & -0.77 & 76.00 & 0.40 \\
\hline Circunvalación (sector este) & 3.50 & -0.56 & 75.68 & 0.27 \\
\hline Rotonda Circunvalación/Avda. La Paz & 3.80 & -0.02 & 75.05 & -0.20 \\
\hline Rotonda Avda. Mendavia/Cicunvalac. & 3.98 & 0.37 & 76.03 & -0.13 \\
\hline Carret. Mendavia (Monte Cantabria) & 3.70 & -0.27 & 76.78 & 0.23 \\
\hline Carret. Mendavia (Pozo Cubillas) & 3.35 & -0.97 & 78.30 & 1.02 \\
\hline Puente Piedra (margen izquierda) & 3.65 & -0.29 & 77.00 & 0.50 \\
\hline Puente Hierro (margen izquierda) & 3.78 & -0.08 & 77.08 & 0.36 \\
\hline Puente Hierro/Sagasta & 4.25 & 0.99 & 75.65 & 0.08 \\
\hline Sagasta/Bretón & 4.73 & 1.99 & 73.53 & -1.02 \\
\hline Fuente Murrieta & 4.18 & 0.81 & 74.75 & -0.40 \\
\hline Gonzalo Berceo/Marqués Murrieta & 4.50 & 1.41 & 74.33 & -0.60 \\
\hline M. Murrieta (Ferrocarril) & 4.03 & 0.44 & 74.40 & -0.61 \\
\hline Yagüe & 3.80 & 0.00 & 75.05 & -0.26 \\
\hline Yagüe (Ulecia) & 3.38 & -0.79 & 77.05 & 0.56 \\
\hline Rotonda Calle Chile & 4.05 & 0.56 & 74.70 & -0.75 \\
\hline Rotonda Avda. Salustiano (Circunv.) & 3.35 & -0.78 & 79.43 & 0.48 \\
\hline Acceso A-68 & 3.05 & -1.42 & 80.68 & 0.84 \\
\hline Lardero (centro pueblo) & 3.65 & -0.23 & 79.28 & 0.39 \\
\hline Lardero/Carretera Soria & 3.33 & -0.87 & 79.08 & 0.45 \\
\hline CC.EE.II. & 3.60 & -0.28 & 79.10 & 0.37 \\
\hline Rotonda Avda. Madrid/Circunval. & 3.88 & 0.26 & 78.20 & 0.02 \\
\hline Promedio & 3.79 & 0.00 & 76.37 & 0.00 \\
\hline Max-min Diferencia & 1.68 & 3.41 & 7.15 & 2.03 \\
\hline
\end{tabular}


La relación existente entre la temperatura y la humedad de una masa de aire es una relación matemática de la que se obtiene el punto de rocío (temperatura a la cual una masa de aire con características higrométricas estables inicia la condensación del vapor de agua). Se acepta la tendencia lógica de que cuanto menor es la temperatura ambiental mayores son la probabilidades de que nos encontremos muy próximos o por debajo del punto de rocío, aumentando con ello los efectos negativos de la humedad en la vida cotidiana.

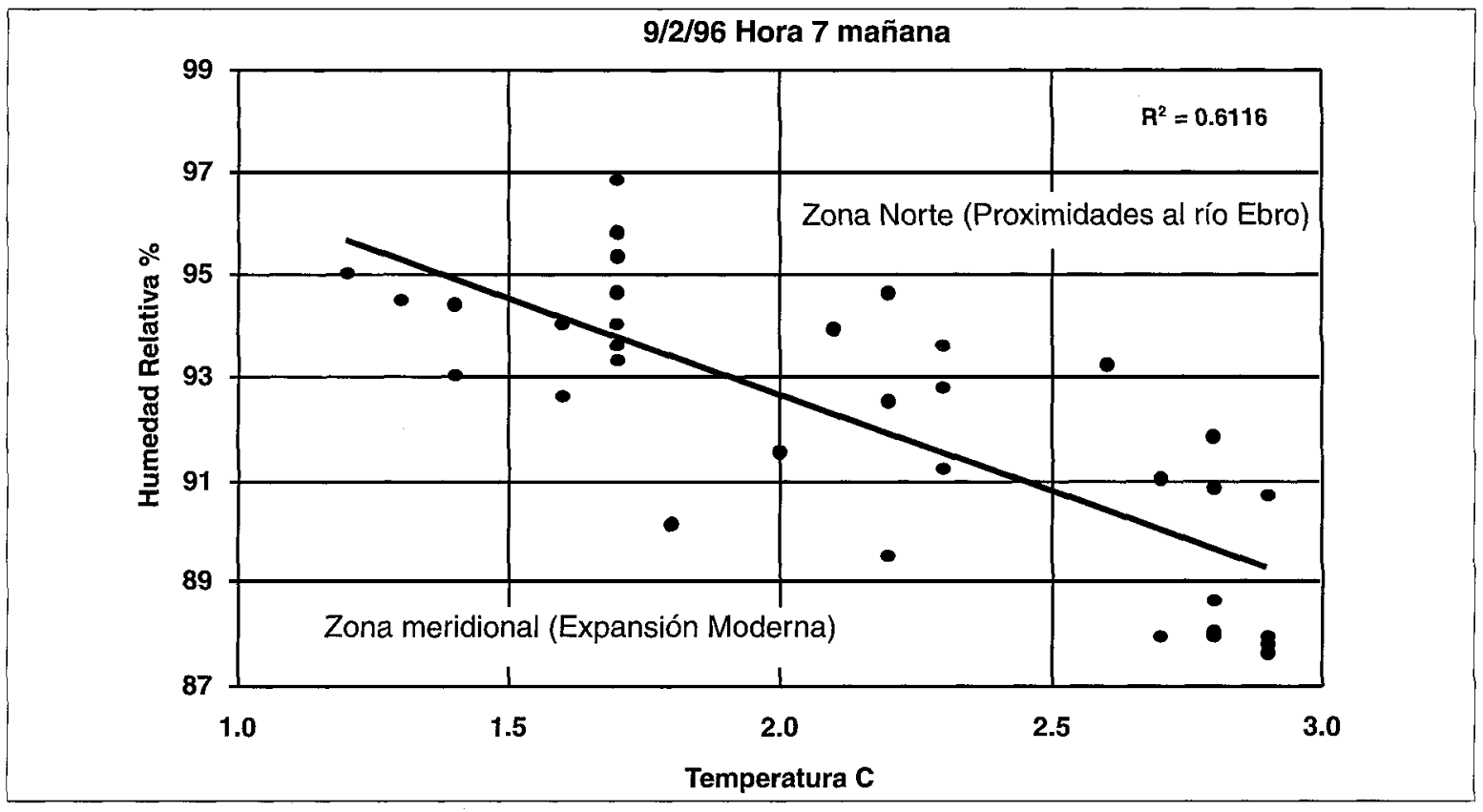

Fig. 5 Relación entre la temperatura y humedad relativa

En la figura 5 se observan ejemplos representativos de la correlación gráfica y numérica existente entre las temperaturas y la humedad relativa en un determinado momento invernal. Los valores varían de un punto a otro de la ciudad y se aprecia bien la tendencia inversa entre estos parámetros climáticos. Interesa resaltar en el gráfico la dispersión de los datos, intuyéndose dos líneas de regresión paralelas entre sí. Efectivamente, existen dos tipos de localizaciones urbanas con diferente comportamiento entre temperatura y humedad (diferencias de un 10\%):

- 1) Los puntos de muestreo localizados en torno al río Ebro (puente de Hierro, ribera izquierda del Ebro, márgenes del casco antiguo, rotonda de la Fuente de Murrieta), y

- 2) El centro y el sector meridional de la ciudad, siempre más secos, aunque sus temperaturas sean incluso más bajas. 
En la Figura 6 se presenta un gráfico descriptivo del índice normalizado de humedad relativa para los puntos de muestreo, realizado a partir de las medias y valores estándar de los muestreos $(x=79,4 \%$, ds $=2,6 \%$ y máxima-míni$\mathrm{ma}=9,4 \%$ ). Se reconoce la mayor humedad del casco antiguo de la ciudad y las riberas del Ebro. Por el contrario, las zonas relativamente más secas de la ciudad (Calvo Sotelo, Avda. Cólon, Jorge Vigón) coinciden con los sectores más alejados del Ebro.

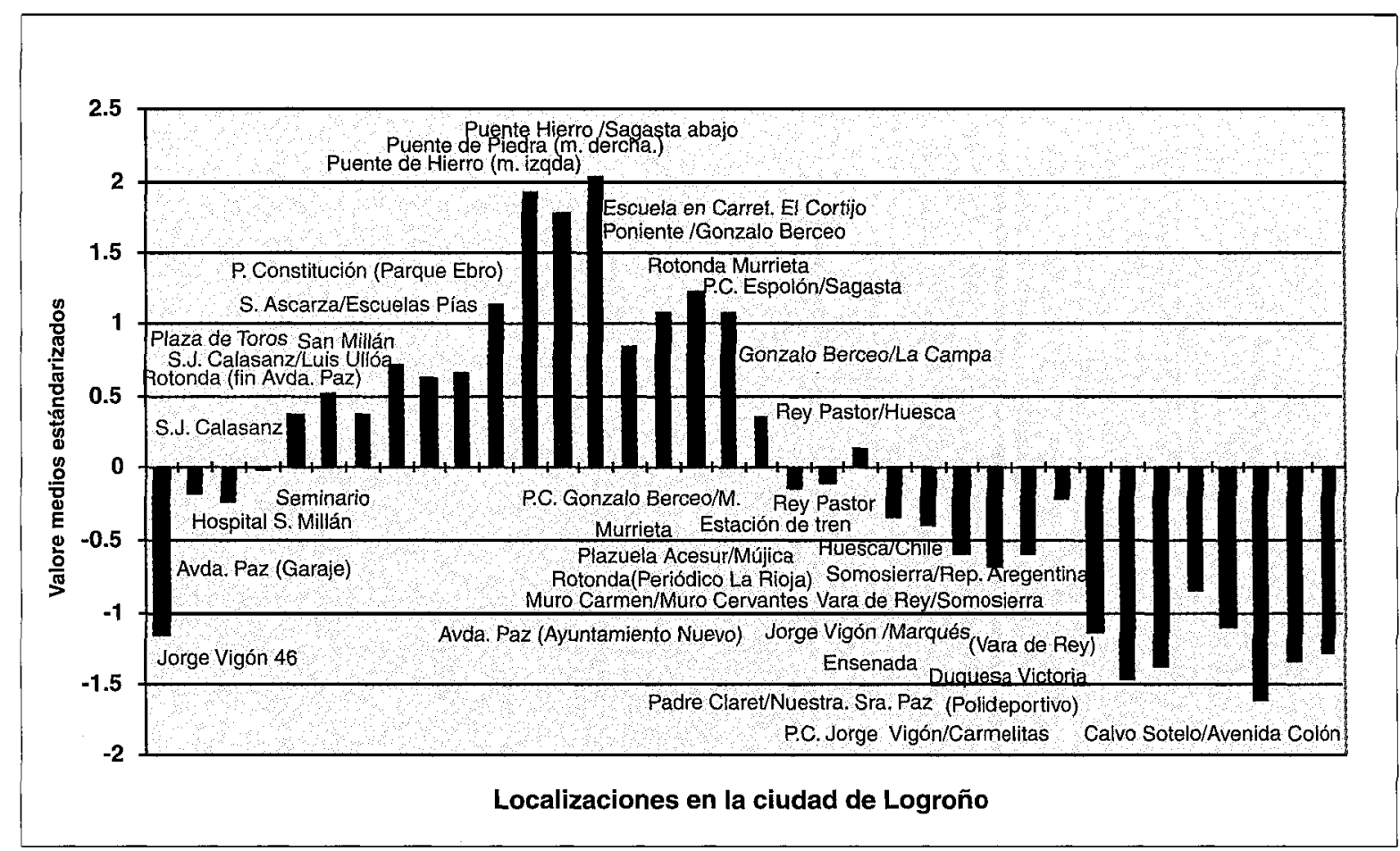

Fig.6 Diferenciacion de la humedad relativa (valores medios estandarizados) en el microclima urbano

\section{Discusión y conclusiones}

A partir de la información presentada se deduce que la ciudad de Logroño ofrece durante el invierno un microclima urbano, con ligera elevación de las temperaturas (isla de calor). Sin embargo se trata de un modesto foco microclimático, ya que las diferencias máximas observadas entre la ciudad y su entorno periférico no superan los $3^{\circ} \mathrm{C}$, y está diferencia se alcanza principalmente en aquellos momentos en que la ciudad irradia energía. El rango de variación térmica entre la ciudad ribereña y sus extremos es aún más reducido y se cifra en $1,7^{\circ} \mathrm{C}$ y en un $10 \%$ de humedad relativa. Estos valores térmicos está muy lejos de los $8^{\circ} \mathrm{C}$ en que se cifran los contrastes máximos de la isla de calor en la metrópoli de Barcelona (Moreno García, 1994). 
La diferenciación térmica observada depende sobre todo de la morfología y las funciones urbanas. En general, el centro, los cruces viales, los barrios residenciales del interior de la ciudad son espacios más cálidos, debido a la protección de los edificios, el tráfico y la irradiación energética. Por el contrario, las zonas verdes, áreas no urbanizadas, y los barrios periféricos son zonas relativamente más frescas. Estos comportamientos térmicos son prácticamente independientes de su distanciamiento al centro de gravedad urbano. Con respecto a la humedad relativa, el río Ebro aporta a la ciudad próxima una mayor humedad ambiental que va disminuyendo a medida que nos alejamos de sus márgenes.

La distribución higrotérmica en la ciudad de Logroño no se ajusta a un modelo espacial de centro-periferia, donde las variaciones se van detectando hacia el exterior urbano. Mas bien, el modelo higrotérmico obedece a una organización multinúcleo, condicionada por la intensidad de la actividad ciudadana y el uso dado al espacio.

\section{Agradecimientos}

Este trabajo se ha realizado con la financiación del proyecto "Optimización de recursos energéticos" (Secretaría de Estado para la Educación)

\section{Referencias bibliográficas}

Almendros, M.A., 1992.- Aspectos climáticos del parque del Retiro (Madrid). Estudios Geográficos, 53 (207): 217-239 pp.

Bello Fuentes, V., 1994.- La isla de calor y los usos del suelo en Guadalajara. Serie Geográfica: El cambio climático y las actividades bumanas, 4: 8398 pp. Universidad de Alcalá.

Brunet, M., 1992.- La magnitud y fluctuaciones de la "isla de calor" en Tarragona. Tarraco, 7: 19-29 pp.

Carreras, C., Marín, M., Martín Vidé, J., Moreno García, M. y Sabi, J., 1990.Modificaciones térmicas en las ciudades. Avance sobre la isla de calor en Barcelona. Documents d' Analisi Geografica, 17: 51-77 pp.

Cuadrat, J.M., Riva, J., López, F. y MarTí, A., 1993a.- El medio ambiente urbano en zaragoza. observaciones sobre la "isla de calor". Anales de Geografía de la Universidad Complutense, 13: 127-138 pp.

Cuadrat, J.M., Riva, J., López, F. y Martí, A., 1993b.- Ciudad y medio ambiente: la isla de calor de Teruel. Geograpbicalia, 30: 113-123 pp.

EASTMAN, J.R., 1995.- Idrisi for Windows. User's Guide version 1.o. Idrisi Production, Clark University. 
Fernández García, F., 1982.- La intensidad del viento en Madrid y sus alrededores. Estudios Geográficos, 166: 17-32 pp. Madrid.

FERnández García, F., 1990.- La influencia de la ciudad sobre las precipitaciones: el caso de Madrid. Estudios Geográficos, 51 (199-200): 397-411 pp.

FERNÁNDEZ García, F., 1994.- Clima y confortabilidad humana. Aspectos metodológicos. Serie Geográfica: El cambio climático y las actividades bumanas, vol. 4: 109-125 pp. Universidad de Alcalá.

García-Ruiz, J.M., Ortigosa, L.M., ARnáez, J. y Gómez, A., 1989.- Organización espacial de las temperaturas en la ciudad de Logroño. Cuadernos de Investigación Geográfica, XV (1-2): 87-89 pp. Logroño.

GoikoetXeA, I., 1991.- El medio ambiente urbano en donostia. observaciones sobre la "isla de calor" generada en el medio urbano. Lurralde. Investigacion y Espacio, 14: 143-162 pp.

GRImmond, C.S. \& Souch, C., 1994.- Surface description for urban climate studies: a GIS based methodology. Geocarto International, 9 (1), $47-59$ pp.

LÓPEZ GÓMEZ, A. (coordinador), 1988.- El clima urbano de Madrid: la isla de calor. Instituto de Economía y Geografía Aplicadas. C.S.I.C., 199 pp. Madrid.

López Gómez, A. (coordinador), 1993.- El clima de las ciudades españolas. Editorial Cátedra, 269 pp. Madrid.

LOWRY, W.P., 1975.- El clima de las ciudades. El Hombre y la Ecosfera. Editorial Blume (Scientific American), 202-210 pp. Madrid-Barcelona.

MARTín VidÉ, J., 1987.- Acerca de la medicion y la experimentacion en geografia fisica. un ejemplo climatologico: la "isla de calor". Notes de Geografia Fisica, 15-16: 21-27 pp.

Moreno García, M., 1993.- Estudio del clima urbano de Barcelona. La "isla de calor". Instituto Nacional de Meteorología, $82 \mathrm{pp}$. Madrid.

Nuñez Olivera, E. y MARTínez AbAigar, J., 1991.- El clima de La Rioja: análisis de precipitaciones y temperaturas.

Riva, J., Cuadrat, J.M., López-Martín, F. y Martí, A., 1997.- Aplicacion de las imagenes Landsat TM al estudio de la isla termica de Zaragoza. Primeros resultados. Geographicalia, 35: 227-242 pp.

SÁNCHEZ GABRIEL, M., 1979.- Climatología y bioclimatología aplicadas a La Rioja. Instituto de Estudios Riojanos, 125 pp. Logroño.

San Pedro Mendoza, F., 1994.- Aproximación al análisis de los climas urbanos sobre series horarias. Serie Geográfica: El cambio climático y las actividades bumanas, vol. 4: 99-106 pp. Universidad de Alcalá.

SPIEGEL, M.R., 1970.- Estadística. McGraw Hill, 357 pp.

SPSS, 1990.- SPSS Reference guide. SPSS Inc., 949 pp. Chicago, USA (ISBN 0918469-62-7). 